\title{
Association of response to hepatitis B vaccination and survival in dialysis patients
}

\author{
Shih-Yi Lin ${ }^{1,2}$, Jiung-Hsiun Liü ${ }^{2,3}$, Shu-Ming Wang ${ }^{2,3}$, I-Kuan Wang ${ }^{1,2,3}$, Chen-An Tsai' ${ }^{4}$ Yao-lung Liu ${ }^{2,3^{*}}$, \\ Hsin-Hung Lin ${ }^{1,2}$, Chiz-Chung Chang ${ }^{2,3}$ and Chiu-Ching Huang ${ }^{2,3}$
}

\begin{abstract}
Background: The status of immunocompromised patients is well recognized in end stage renal disease (ESRD). As described recently, this acquired immune dysfunction in the uremic milieu may be one of the main pathogenic factors for mortality in ESRD. The aim of this study was to determine the relationship between the immune response following a hepatitis $B$ vaccination (HBV vaccination) and the survival of maintenance dialysis patients.

Methods: A total of 156 patients (103 on hemodialysis and 53 on continuous ambulatory peritoneal dialysis) were recruited. After receiving a full dose of the HBV vaccination, all patients were followed up for to 5 years to evaluate the association of patient survival, cause of mortality, and immune response.

Results: The response rate to the hepatitis B vaccination was $70.5 \%$. There was no significant association between the immune response and the 5 -year survival rate $(p=0.600)$ or between the post-vaccination anti-HBs titers and the 5 -year survival rate $(p=0.201)$. The logistic prediction model with the coefficient as non-response following HBV vaccination, diabetes mellitus, old age, and low albumin level could significantly predict infection-cause mortality (sensitivity $=0.842$, specificity $=0.937$ ).

Conclusion: There was no significant association between the immune response to HBV vaccination and the 5-year survival rate. However, non-response following HBV vaccination might be associated with infection-cause mortality in dialysis patients.
\end{abstract}

Keywords: Hepatitis B vaccination, Immune response, Post-vaccination anti-HBs titers

\section{Background}

End-stage renal disease (ESRD) significantly increases the risk of mortality and morbidity. The leading cause of death in ESRD is cardiovascular disease (CVD), which accounts for more than half of deaths in uremic patients, with infection the second leading cause [1,2]. Previous studies have investigated the relationships among endothelial dysfunction, inflammation, and atherosclerotic vascular disease in chronic kidney disease [3,4]. Uremicrelated inflammation, which commonly occurs during pre-dialysis and persists even after dialysis, is now regarded as an important predictor of cardiovascular and all-cause mortality in the ESRD population [5].

\footnotetext{
* Correspondence: liuyaolung@yahoo.com

${ }^{2}$ Division of Nephrology and Kidney institute, China Medical University Hospital, Taichung, Taiwan

${ }^{3}$ School of Medicine, China Medical University, Taichung, Taiwan

Full list of author information is available at the end of the article
}

Currently, it is speculated that acquired immune dysfunction in the uremic milieu may be one of the main pathogenic factors, mediated through CVD and infections, in most ESRD-related deaths. Concomitant immunosuppression and immune activation in uremia would predispose patients to infection and CVD, respectively [6].

Although it is well established that aging, diabetes mellitus (DM), and the presence of co morbid conditions predict a poor prognosis in ESRD, few studies have investigated immune dysregulation as a determinant of mortality in ESRD [7]. Furthermore, acquired immune dysfunction in uremic patients has various clinical features, ranging from proness to infection to impaired immune response to vaccinations. The aim of our study was to link the clinical immune parameter, immune response and anti-HBs titers one month following HBV vaccination with mortality in ESRD populations.

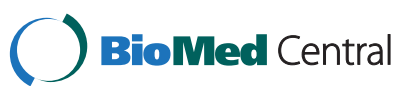

(c) 2012 Lin et al.; licensee BioMed Central Ltd. This is an Open Access article distributed under the terms of the Creative Commons Attribution License (http://creativecommons.org/licenses/by/2.0), which permits unrestricted use, distribution, and reproduction in any medium, provided the original work is properly cited. 


\section{Methods}

This clinical study complied with the Declaration of Helsinki and was approved by the Medical Ethics Committee of the China Medical University Hospital, Taichung (Taiwan, ROC).

This was a retrospective study of ESRD patients who underwent dialysis therapy at the dialysis unit of the China Medical University Hospital (Taichung, Taiwan) from March 2002 to March 2008 and who were followed up until March 2009.

The HBV vaccine was offered to all patients who tested negative for the HBV surface antigen (HBs Ag) and anti-HBs and who had not previously received the HBV vaccination. We excluded patients who had malignancies, who were receiving immunosuppressive agents, or who did not receive all four vaccinations. All patients were given four doses ( $40 \mu \mathrm{g}$ per dose) of the hepatitis B vaccine (Engerix-B, GlaxoSmithKline Biologicals, Philadelphia, USA) in the deltoid muscles at $0,1,2$, and 6 months. Anti-HBs titers were measured by an ELISA kit (AUSAB-EIA, Abbot Labs, Chicago, USA) one month after the final dose and annually thereafter.

We defined responders and non-responders according to the level of anti-HBs one month after the final injection (non-responders: < $10 \mathrm{IU} / \mathrm{L}$; responders: $\geq 10 \mathrm{IU} / \mathrm{L}$ ). These patients were followed up to assess the relationship between the initial immune response to vaccination and the 5-year-survival. In addition, over the 5-year follow-up period, we assessed the effect of one-month post-vaccination anti-HBs titers on survival.

To determine the factors related to HBs antibody formation following vaccination, we recorded the patient age, gender, presence of diabetes mellitus (DM), hemoglobin, serum albumin, triglyceride, cholesterol, fasting blood sugar, presence of hepatitis $\mathrm{C}$ antibodies, and dialysis modality at the initiation of vaccination [8-10].

\section{Statistical analysis}

All statistical tests were performed with SPSS (version 15.0). A P value $<0.05$ was considered statistically significant. For categorical variables, we used the Chi-squared test and presented data as absolute numbers or percentages. For continuous variables, we used Student's $t$-test and presented the data as means \pm standard deviations. The Cox regression model was used to investigate the association between seroconversion and mortality and the relationship between post-vaccination anti-HBs titers and mortality.

We adjusted for age, level of albumin, presence of DM, dialysis modality, and gender to determine the adjusted hazard ratios with the immune response to mortality.

We adapted the logistic regression model as follows: $\beta 0+\beta 1 \%$ Non-response $+\beta 2 \%($ presence of $\mathrm{DM})+$ $\beta 3 *$ Age $+\beta 4 *$ Albumin $+\beta 5 \%$ (presence of DM)*Albumin + $\beta 6^{*}$ Age $e^{*}$ Albumin. We used this equation to evaluate the effects of non-response, diabetes mellitus, age, and the level of albumin in predicting mortality due to infection.

\section{Results}

A total of 156 patients (64 males, 92 females) were recruited, with 110 responders and 46 non-responders (Table 1). Mean ages were higher for non-responders $(62.63 \pm 9.95$ years $)$ than for responders $(55.75 \pm 14.31$ years, $\mathrm{P}=0.003)$. The prevalence of diabetes or anti-HBV vaccination at the start of the study was $32.0 \%$.

Univariate analysis indicated no significant differences between responders and non-responders in terms of gender, presence of DM, presence of hepatitis C antibodies, hemoglobin, albumin, dialysis modality, triglyceride, cholesterol, and several other relevant factors (Table 1). By the time of the 5-year follow up, 35 patients had died. The most common cause of death was infection (54.3\%; 19 patients, including 8 cases of severe pneumonia, 4 cases of peritonitis, 2 cases of infective endocarditis, and 1 case of either meningitis, diabetic foot infection, septic arthritis, urosepsis, and septicemia), followed by cardiovascular events (25.7\%, 9 patients), gastrointestinal bleeding (5.7\%, 2 patients), gastrointestinal perforation $(5.7 \%$, 2 patients), and others (8.6\%, 3 patients). By the end of the study, the prevalence of DM in all available subjects (including those that died during the study) was 30\%, but the impact of DM on the loss of anti-HBs Ab was not significant $(\mathrm{p}=0.308)$.

We followed up on all patients after the vaccination to investigate the association between seroconversion and mortality. Figure 1 shows that there was no significant survival difference for responders and non-responders $(P=0.297)$. A Cox regression analysis, which considered patient age, presence of DM, initial serum albumin

Table 1 Baseline characteristics of HBV vaccine responders and non-responders

\begin{tabular}{llll}
\hline Characteristic & Non-responders & Responders & P value \\
\hline Number of pts. & 46 & 110 & \\
Age & $62.63 \pm 9.95$ & $55.75 \pm 14.31$ & 0.003 \\
Gender(M/F) & $17 / 29$ & $47 / 63$ & 0.507 \\
DM & 19 & 31 & 0.111 \\
HCV infection & $9(19.6 \%)$ & $15(13.6 \%)$ & 0.353 \\
Hemoglobin(g/dL) & $9.49 \pm 1.42$ & $9.55 \pm 1.38$ & 0.786 \\
Albumin(g/dL) & $3.47 \pm 0.37$ & $3.50 \pm 0.39$ & 0.696 \\
Dialysis modality (HD:PD) & $31: 15$ & $72: 38$ & 0.817 \\
Cholesterol(mg/dL) & $184.71 \pm 50.07$ & $181.33 \pm 42.66$ & 0.669 \\
Triglyceride(mg/dL) & $187.93 \pm 131.87$ & $176.58 \pm 124.46$ & 0.610 \\
\hline Note: data are shown as the & number &
\end{tabular}

Note: data are shown as the number (\%) or mean $\pm \mathrm{SD}$, as appropriate. Abbreviations: $M$, male; $F$, female. 


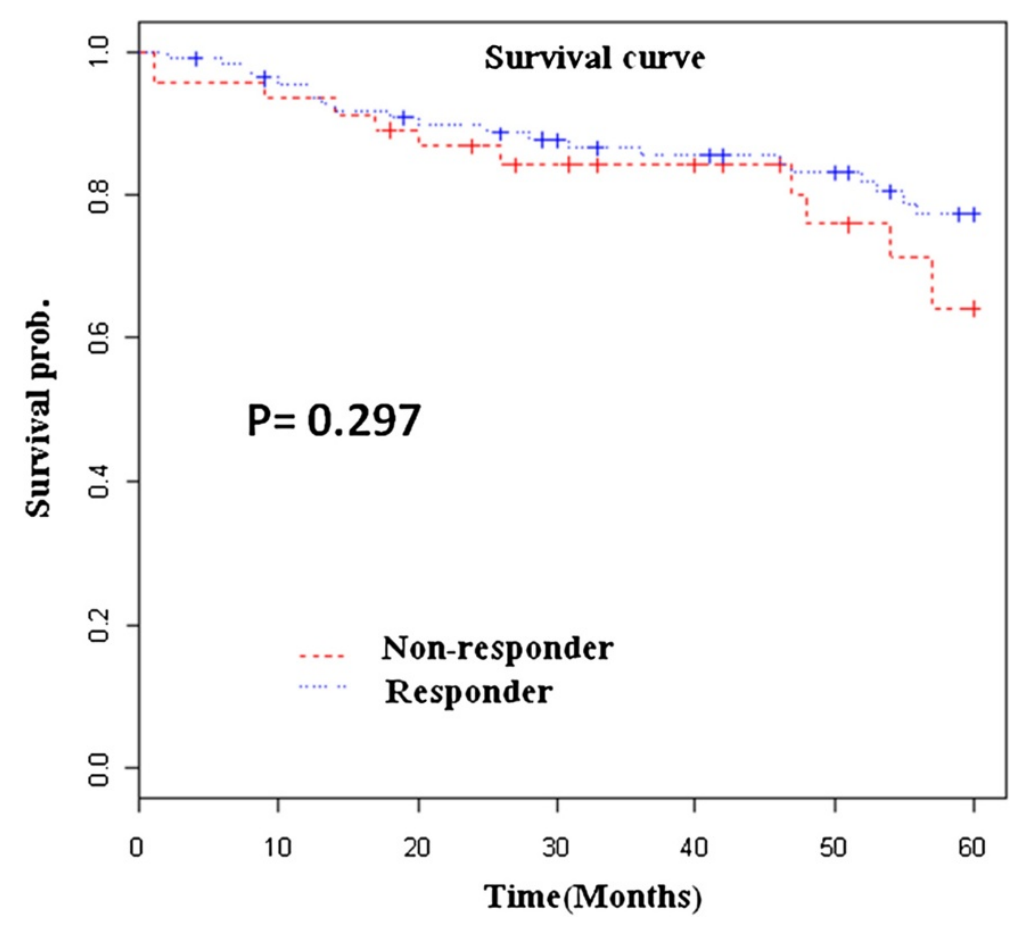

Figure 1 Cumulative survival of HBV vaccine responders and non-responders over 5 years.

levels, seroconversion after HBV vaccination, dialysis modality, and gender, showed that advanced age and malnutrition were associated with increased mortality $(\mathrm{P}=0.035$ and $\mathrm{P}=0.032$, respectively) (Table 2$)$.

We also evaluated the association between one-month post-vaccination anti-HBs titers and mortality. There was no significant survival difference between immune sustainers and non-sustainers $(\mathrm{P}=0.201)$. Cox regression analysis indicated that only age and low levels of albumin were associated with mortality $(\mathrm{p}=0.04$ and $\mathrm{p}=0.032$, respectively).

The predictive model of mortality due to infection in dialysis patients demonstrated that non-response, DM, age, and low levels of albumin all predicted significantly higher mortality rates due to infection (Table 3). This model performs well and has high sensitivity and high specificity (sensitivity $=0.842$, specificity $=0.938$, positive predictive values $=0.941$, negative predictive value $=$

Table 2 Cox proportional hazards regression of immune response to $\mathrm{HBV}$ vaccination and mortality

\begin{tabular}{lc}
\hline Parameter & P- value \\
\hline Age & 0.035 \\
Modality & 0.210 \\
Seroconversion of HBV vaccination & 0.600 \\
Albumin & 0.032 \\
Gender & 0.060 \\
Diabetes Mellitus & 0.670 \\
\hline
\end{tabular}

0.833). The area under the ROC curve (AUC) provides an overall measure of the model's classification accuracy, with a value of one representing perfect accuracy. The ROC curve shows a high capacity for discriminating infection-cause mortality vs. non-infection cause mortality, with an $\mathrm{AUC}=0.9112$ (Figure 2). The immune response following $\mathrm{HBV}$ vaccination and other co-factors were not associated with CV mortality (data not shown).

\section{Discussion}

In this study, we showed that the seroconversion rate of our dialysis patients was $70.5 \%$ after four double doses (40 $\mu \mathrm{g}$ per dose) of the hepatitis B vaccine administered over 6 months. We also found that responders were

Table 3 The predictive model of infection-cause mortality in dialysis patients Logit (probability of infection-cause mortality) $=\beta 0+\beta 1 *$ Retiter $+\beta 2 * D M+\beta 3^{*} A g e+$ $\beta 4 *$ Albumin $+\beta 5 * D M * A$ Ibumin $+\beta 6 *$ Age*Albumin

\begin{tabular}{lcc}
\hline Covariates & Coefficient & p-value \\
\hline Intercept & 143.4122 & 0.0232 \\
Seroconversion & -4.7392 & 0.0068 \\
following & & \\
HBV vaccination & -5.3569 & \\
Presence of DM & -1.9758 & 0.0084 \\
Age & -40.2689 & 0.3333 \\
Albumin & 13.1720 & 0.0234 \\
DM*Albumin & 0.5699 & 0.0089 \\
Age*Albumin & & 0.0317 \\
\hline
\end{tabular}




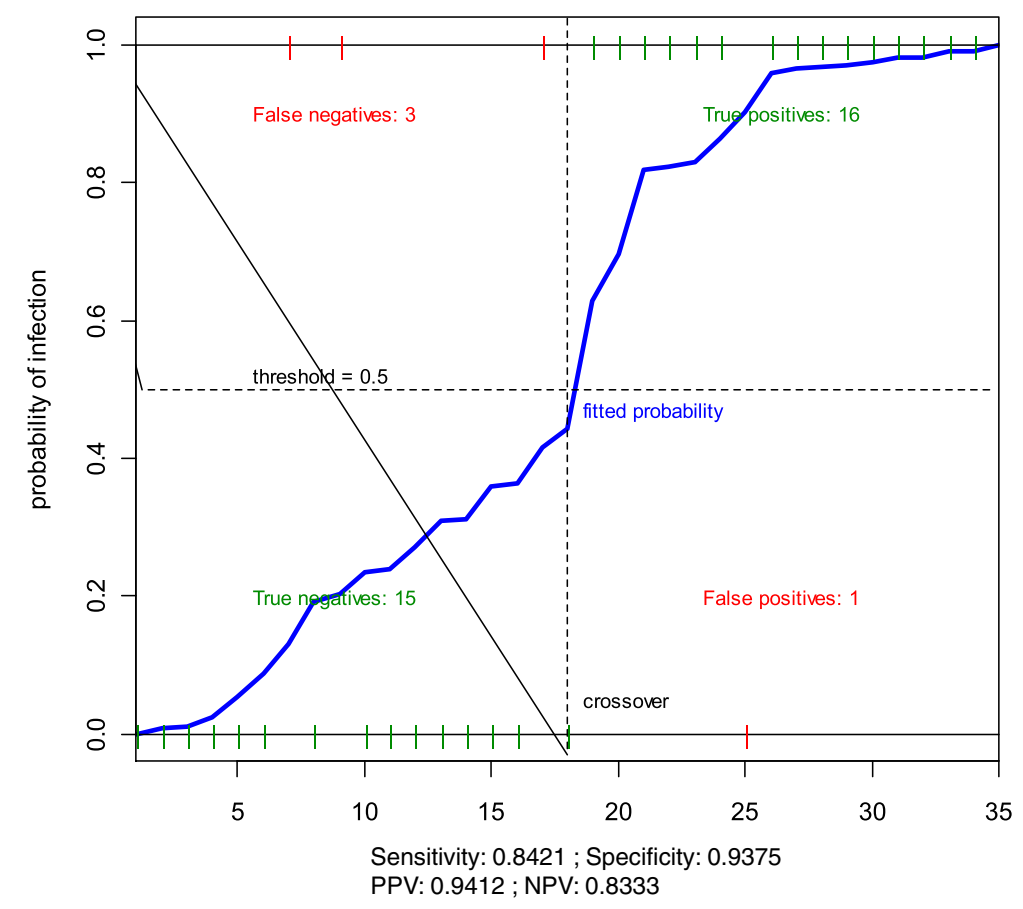

Figure 2 The ROC curve shown used to examine the performance of logistic regression model.

significantly younger than non-responders $(P=0.003)$. Seroconversion to HBV vaccination and one-month post-vaccination anti-HBs titers did not predict all-cause mortality in our maintenance dialysis patients. After adjusting for age, DM, albumin levels, gender, and dialysis modality, non-seroconversion following HBV vaccination could independently predict mortality due to infection in ESRD dialysis patients. This novel and interesting finding demonstrates the possible relationship between the vaccination response and the specific cause of mortality in ESRD patients.

Our study dialysis subjects displayed a similar suboptimal immune response to patients in previous studies. In ESRD patients, the response rate to $\mathrm{HBV}$ vaccination is approximately $50 \%-80 \%$, which is lower than the $90 \%$ response rate in the general population, with advanced age being a significant negative factor associated with this poor immune response [11-13]. Numerous factors associated with the impaired immune response following HBV vaccination in ESRD, such as age, albumin levels, HCV infection, and gender, have been well studied $[8-10,13]$. Current studies have focused on the insufficient acquired immunity due to uremia to account for the suboptimal response rates following HBV vaccination [14]. Recent studies have proposed that the immunodeficiency in ESRD results from the impaired function of antigenpresenting cells, alteration of toll-like receptors, weakened activation of $\mathrm{T}$ lymphocytes, and the imbalance of $\mathrm{T}$ helper lymphocytes (Th 1/Th 2 ratio) [14-16].
In the present study, we demonstrated that DM, age, low albumin levels, and non-response following HBV vaccination could predict mortality due to infection.

Several negative determinants of mortality in ESRD, such as old age, DM, and malnutrition, have been documented [7]. Immune dysfunction in uremia is also recognized as a determinant of mortality. However, our findings are important because they demonstrate a link between infection-cause mortality and insufficient immune responses to HBV vaccination in ESRD, since these two clinical presentations involve homogenous but varied interactions between the immune, innate immune and the adaptive immune systems. A recent review indicated that in dialysis patients innate immunity plays an important role in fighting infections, while acquired immunity (via antibody production) also makes a significant contribution [14]. We therefore hypothesize that $\mathrm{T}$ cell dysfunction may be the main link between the high mortality due to infection and the insufficient antibody response to vaccination in ESRD patients. Impaired cytotoxic CD8 $\mathrm{T}$ cell response limits the fight of the infected host defense system against the pathogens, while the inadequate function of helper CD4 T cells weakens the humoral immune system. The results of studies by Alvarez-lara et al. and Meier P et al. support this hypothesis, finding increased apoptosis of $\mathrm{T}$ cells in uremia [17-19]. An alternative interpretation is that the key linking factor between high mortality due to infection and the impaired vaccination response lies upstream 
of T-cell activation, i.e., the pattern of recognition and the antigen presenting cell or secondary dysregulation or activation of cytokines $[6,14,20]$.

It has been proposed that both the innate and acquired immune systems may play a role in the progression of atherosclerosis, the major pathogenic factor responsible for most deaths in ESRD patients [21]. Interestingly, we did not find an association between seroconversion and all-cause mortality or between seroconversion and CVcause mortality in dialysis patients. The correlations among persistent inflammation, CVD, immune dysfunctions, and infections in ESRD have become apparent in recent years [5,6,22-24].

Chronic inflammation as a result of hypercytokinemia and an imbalance in the pro-inflammatory to antiinflammatory ratio has been well recognized in ESRD [25]. The dysregulation of various cytokines due to the uremia milieu may link inflammation-atherosclerosis to impaired immunity inflammation in dialysis patients [14]. Several studies have attempted to link the levels and types of cytokines with clinical outcomes. Girndt et al. determined that higher levels of IL- 6 and TNF- $\alpha$ correlated with an immunocompromised status, that is, non-seroconversion to $\mathrm{HBV}$ vaccinations in $\mathrm{HD}$ patients [26]. Kimmel et al. assessed immune parameters such as cytokines in $230 \mathrm{HD}$ patients, with a three-year follow up. Their results showed that increased levels of IL-6, TNF- $\alpha$, IL-1, and IL-13 correlated with a higher relative mortality risk. High levels of IL-2, IL-4, IL-5, and IL-12, and $\mathrm{T}$ cell functions were associated with survival in HD patients. The authors did not analyze the causes of mortality in their patients or further investigate the associations between the causes of mortality and cytokines. Interestingly, they noted that the patterns of cytokines involved may be more important than the individual cytokine levels in interpreting survival outcomes [27].

Among the other studies on cytokines' ability to predict mortality in ESRD, the study by Badiou et al. measured levels of both pro-inflammatory cytokines and anti-inflammatory cytokines in $134 \mathrm{HD}$ patients and found that IL-6 levels could strongly predict CV mortality. In addition, (IL-4+ IL-6+ IL-10)/(IL-2 + IFN- $\gamma$ ) was associated with non-CV mortality. The authors speculated that examination of the Th1/Th2 cytokine relation might be more relevant for predicting non-CV mortality [28]. These findings indicate that the laboratory immune parameter, especially the Th1/Th2 cytokine ratio that implies defective immunity, may be able to predict clinical outcomes. The clinical immune parameters and the immune response following HBV vaccination may be able to correlate with mortality in ESRD patients.

The study by Fernandez et al. of 64 HD patients noted that low levels of albumin negatively influenced the response to HBV vaccination. In the study's survival analysis of 31 patients, non-responders had higher mortality and morbidity [29]. The authors did not adjust for albumin levels or age in the survival analysis of these HD subjects. However, our Cox regression survival analysis of 156 dialysis patients reveals that only old age and low levels of albumin have significantly higher mortality but that non-response following HBV vaccination did not. Since low albumin levels are recognized as an independent factor of mortality in ESRD [30], we speculate that low albumin levels account for the concomitant impaired immune response following HBV vaccination and increased mortality in the Fernandez et al. study.

The Kimmel et al. study suggests that better $\mathrm{T}$ cell function and humoral immunity are associated with a survival advantage in HD patients [27]. Unlike the predictive value of the cytokine ratio on mortality mentioned above, our study demonstrated no significant relationship between the clinical immune response following HBV vaccination and all-cause mortality. The levels and types of cytokines in dialysis patients were the result of the interacting influences of residual renal function, dialysis adequacy, comorbidity, and current treatment [31]. Our dialysis patients received HBV vaccinations at the initiation of renal replacement therapy. Some of our study subjects, especially the PD patients, retained some residual renal function, which would affect the immune response to HBV vaccinations. In the Badiou et al. and Kimmel et al. studies, the levels of circulating cytokines (either Th1 or Th2-related) were significantly higher in the HD patients than in the control patients $[27,28]$. The effects of these unusual levels of circulating cytokines may not correlate well with their baseline local immunological functions [31]. These circulating cytokines may operate in a more sophisticated pathophysiologic network, causing erythropoietin resistance, hypoalbuminemia, frailty, atherosclerosis, and dyslipidemia [31,32]. Therefore, the clinical outcomes, survival and immune response following HBV vaccination may be the diverse results of the differing interactions of various cytokines at specific cellular, organ, and system targets. We therefore could not determine whether a direct association exists between seroconversion and all-cause mortality in dialysis patients despite the well-recognized immunodeficiency, hypercytokinemia, and high mortality in ESRD populations [1,14,24,33,34].

We did not check the antibody of the core HBV antigen (anti-HBc). The finding of a positive anti-HBc in the absence of $\mathrm{HBs} \mathrm{Ag}$ or anti-HBs is relatively uncommon [35]. Chen et al. found that the responsiveness rates of the hepatitis $B$ vaccination were the same between isolated anti-HBc positive and normal subjects [36].

We recognize that our study has certain limitations, such as the relatively small number of dialysis patients 
included to determine the infection-based and CV mortality. Moreover, infection was the leading cause of mortality among our study subjects, despite being the second leading cause of deaths in the ESRD population as a whole. The immune status of dialysis patients may change after losing residual renal function. The results of our study need to be replicated in prospective studies of using larger populations of chronic, stable dialysis patients.

\section{Conclusions}

In conclusion, we demonstrated that non-response following HBV vaccination might be associated with infection-cause mortality in our dialysis patients. However, the clinical immune response following HBV vaccination would not be associated with all-cause mortality in our dialysis patients.

\section{Competing interests}

The authors declare that they have no competing interests.

\section{Authors' contributions}

SL carried out the data collection and drafted the manuscript. JL, SW, and I W participated in the design of the study. $\mathrm{CT}, \mathrm{HL}, \mathrm{CC}$, and $\mathrm{CH}$ performed the statistical analysis. YL conceived of the study, and participated in its design and coordination. All authors read and approved the final manuscript.

\section{Author details}

${ }^{1}$ Graduate Institute of Clinical Medical Science, College of Medicine, China Medical University, Taichung, Taiwan. ${ }^{2}$ Division of Nephrology and Kidney institute, China Medical University Hospital, Taichung, Taiwan. ${ }^{3}$ School of Medicine, China Medical University, Taichung, Taiwan. ${ }^{4}$ Department of Agronomy, National Taiwan University, Taipei, Taiwan.

Received: 24 October 2011 Accepted: 27 August 2012

Published: 30 August 2012

\section{References}

1. Collins AJ: Cardiovascular mortality in end-stage renal disease. Am J Med Sci 2003, 325:163-167.

2. Sarnak MJ, Laber BL: Mortality caused by sepsis in patients with end-stage renal disease compared with the general population. Kidney Int 2000, 58:1758-1764

3. Cleland SJ, Sattar N, Petrie JR, Forouhi NG, Elliott HL, Connell JM: Endothelial dysfunction as a possible link between C-reactive protein levels and cardiovascular disease. Clin Sci 2000, 98:531-535.

4. Raza K, Thambyrajah J, Townend JN, Exley AR, Hortas C, Filer A, Carruthers DM, Bacon PA: Suppression of inflammation in primary systemic vasculitis restores vascular endothelial function: lessons for atherosclerotic disease? Circulation 2000, 102:1470-1472

5. Zimmermann J, Herrlinger S, Pruy A, Metzger T, Wanner C: Inflammation enhances cardiovascular risk and mortality in hemodialysis patients. Kidney Int 1999, 55:648-658.

6. Kato S, Chmielewski M, Honda H, Pecoits-Filho R, Matsuo S, Yuzawa Y, Tranaeus A, Stenvinkel P, Lindholm B: Aspects of immune dysfunction in end-stage renal disease. Clin J Am Soc Nephrol 2008, 3:1526-1533.

7. Vonesh EF, Snyder JJ, Foley RN, Collins AJ: The differential impact of risk factors on mortality in hemodialysis and peritoneal dialysis. Kidney Int 2004, 66:2389-2401.

8. Peces R, de la Torre M, Alcázar R, Urra JM: Prospective analysis of the factors influencing the antibody response to hepatitis $B$ vaccine in hemodialysis patients. Am J Kidney Dis 1997, 29:239-245.

9. Navarro JF, Teruel JL, Mateos M, Ortuno J: Hepatitis C virus infection decreases the effective antibody response to hepatitis $B$ vaccine in hemodialysis patients. Clin Nephrol 1994, 41:113-116.
10. Liu YL, Kao MT, Huang CC: A comparison of responsiveness to hepatitis $B$ vaccination in patients on hemodialysis and peritoneal dialysis. Vaccine 2005, 23:3957-3960.

11. Poland GA, Jacobson RM: Prevention of hepatitis $B$ with the hepatitis $B$ vaccine. N Eng J Med 2004, 351:2832-2833.

12. Stevens CE, Alter HJ, Taylor PE, Zang EA, Harley EJ, Szmuness W: The dialysis vaccine trial study group. N Engl I Med 1984, 311:496-501.

13. Fabrizi F, Martin P, Dixit V, Bunnapradist S, Dulai G: Meta-analysis: the effect of age on immunological response to hepatitis $B$ vaccine in end-stage renal disease. Aliment Pharmacol Ther 2004, 20:1053-1062.

14. Eleftheriadis T, Antoniadi G, Liakopoulos V, Kartsios C, Stefanidis I: Disturbances of acquired immunity in hemodialysis patients. Semin Dial 2007, 20:440-451.

15. Ando M, Shibuya A, Yasuda M, Azuma N, Tsuchiya K, Akiba T, Nitta K: Impairment of innate cellular response to in vitro stimuli in patients on continuous ambulatory peritoneal dialysis. Nephrol Dial Transplant 2005, 20:2497-2503.

16. Sester U, Sester M, Hauk M, Kaul H, Köhler H, Girndt M: T-cell activation follows Th1 rather than Th2 pattern in haemodialysis patients. Nephrol Dial Transplant 2000, 15:1217-1223.

17. Alvarez-Lara MA, Carracedo J, Ramírez R, Martín-Malo A, Rodríguez M, Madueño JA, Aljama P: The imbalance in the ratio of Th1 and Th2 helper lymphocytes in uremia is medicated by an increased apoptosis of Th1 subset. Nephrol Dial Transplant 2004, 19:3084-3090.

18. Meier P, Dayer E, Blanc E, Wauters JP: Early T cell activation correlates with expression of apoptosis markers in patients with end-stage renal disease. J Am Soc Nephrol 2002, 13:204-212.

19. Litjens NH, Van Druningen CJ, Betjes MG: Progressive loss of renal function is associated with the activation and depletion of naïve T lymphocytes. Clin Immunol 2006, 118:83-91.

20. Stenvinkel P, Ketteler M, Johnson RJ, Lindhom B, Pecoits-Filho R, Riella M, Heimburger O, Cederholm T, Girndt M: IL-10, IL-6, and TNF-a: central factors in the altered cytokine network of uremia- the good, the bad, and the ugly. Kidney Int 2005, 67:1216-1233.

21. Binder CJ, Chang MK, Shaw PX, Miller YI, Hartvigsen K, Dewan A, Witztum JL: Innate and acquired immunity in atherogenesis. Nat Med 2001, 8:1210-1226.

22. Jofre R, Rodriguez-Benitez P, Lopez-Gomez JM, Perez-Garcia R: Inflammatory syndrome in patients on hemodialysis. J Am Soc Nephrol 2006, 17(suppl 3):S274-S280.

23. Pecoits-Filho R, Bárány $P$, Lindholm B, Heimbürger O, Stenvinkel P: Interleukin-6 is an independent predictor of mortality in patients staring dialysis treatment. Nephrol Dial Transplant 2002, 17:1684-1688.

24. Kaysen GA: The microinflamatory state in uremia: causes and potential consequences. J Am Soc Nephrol 2001, 12:1549-1557.

25. Girndt M, Ulrich C, Kaul H, Sester U, Sester M, Köhler H: Uremia-associated immune defect: The IL-10-CRP axis. Kidney Int 2003, 63:S76-S79.

26. Girndt M, Kohler H, Schiedheim-Weick E, Schlaak JF, Zumbuschenfelde K-HM, Fleischer B: Production of interleukin-6, tumor necrosis factor- $a$ and interleukin-10 in vitro correlate with the clinical immune defect in chronic hemodialysis patients. Kidney Int 1995, 47:559-565.

27. Kimmel PL, Phillips TM, Simmens SJ, Petersons RA, Weihs KL, Alleyne S, Cruz I, Yanovski JA, Veis JH: Immunological function and survival in hemodialysis patients. Kidney Int 1998, 54:236-244.

28. Badiou S, Cristol JP, Jaussent I, Terrier N, Morena M, Maurice F, LerayMorageus H, Rivory JP, Chalabi L, Delcourt C, Canaud B, Dupuy AM: Fine tuning of the prediction of mortality in hemodialysis patients by use of cytokine protemic determintation. Clin J Am Soc Nephrol 2008, 3:423-430

29. Fernandez E, Betriu MA, Gómez R, Montoliu J: Response to the hepatitis B virus vaccine in haemodialysis patients: influence of malnutrition and its importance as a risk factor for morbidity and mortality. Nephrol Dial Transplant 1996, 12:1204-1211.

30. Lowrie EG, Lew NL: Death risk in hemodialysis patients: the predictive value of commonly measured variables and an evaluation of death rate differences between facilities. Am J Kidney Dis 1990, 15:458-482.

31. Dinarello CA: Historical insights into cytokines. Eur J Immunol 2007, 37:S34-S45.

32. Tedgui A, Mallat Z: Cytokines in atherosclerosis: pathogenic and regulatory pathways. Physiol Rev 2006, 86:515-581.

33. Kaysen GA: Biochemistry and biomarkers of inflamed patients: why look, what to assess. Clin J Am Soc Nephrol 2009, 4:S56-S63. 
34. Dinarello CA: Cytokines: agents provocateurs in hemodialysis? Kidney Int 1992, 41:683-694.

35. Davis AR, Pink JM, Lien AH, Wylie BR: Isolated antibody to hepatitis B core in blood donors and immune response to hepatitis B vaccination. Aust $N$ ZJ Med 1993, 23:215-233.

36. Chan CY, Lee SD, Tasi YT, Lo KJ: Hepatitis B vaccination alone is not adequate for the categorizing of adult subjects with isolated anti-HBc. J Gastroenterol Hepatol 1995, 10:192-197.

doi:10.1186/1471-2369-13-97

Cite this article as: Lin et al: Association of response to hepatitis B vaccination and survival in dialysis patients. BMC Nephrology 2012 13:97.

\section{Submit your next manuscript to BioMed Central and take full advantage of:}

- Convenient online submission

- Thorough peer review

- No space constraints or color figure charges

- Immediate publication on acceptance

- Inclusion in PubMed, CAS, Scopus and Google Scholar

- Research which is freely available for redistribution 